\title{
Foam Washout Sclerotherapy, a single center experience
}

\author{
K Fattahi ${ }^{1}$ \\ ${ }^{1}$ Vein Specialty Medical Clinic Inc., Campbell, CA USA
}

submitted: Jan 4, 2020, accepted: Jul 15, 2020, EPub Ahead of Print: Aug 12, 2020

Conflict of interest: None

DOI: 10.24019/jtavr.82 - Corresponding author:Dr. Khalil Fattahi, kfparsi@gmail.com

(C) 2018 Fondazione Vasculab impresa sociale ONLUS. All rights reserved.

\begin{abstract}
Introduction: The author has designed and implemented a modified method of foam sclerotherapy (FS) over some years which he believes lowers the rate of complications of the procedure while maintaining efficacy of the procedure as assessed clinically and by duplex ultrasound. This modified technique is termed Foam Washout Sclerotherapy (FWS) and involves the removal of the injected foam from a point distal to its administration level. This article compares the success rate and the rate of complications between FWS and FS of varicose veins. All procedures have been performed by the author over a period of 4 years. The basic principle of FWS is to inject a certain volume of a detergent-based foamed medication at the proximal part of the targeted vein and removing the injected foam from the same vein via a distally placed IV catheter within 5-10 seconds. The primary end point of the study was limited only to the comparison of the closure rate of treated vein segments (success rate) and complication rates.

Materials and Methods: A retrospective review of FWS and FS cases performed between late 2008 to late 2012 was carried out. The review included only the cases that had regular follow-ups for one year consisting of 823 sclerotherapy treatments (292 in FS group and 531 in FWS group) among 657 patients. Studied veins were great saphenous (GSV), small saphenous (SSV), and anterior accessory saphenous vein (AASV) varicosities. Follow up included a phone call in 24 hours, re-exam and ultrasound studies in 2 months, 6 months, and 12 months. Most patients had additional follow up in 2-3 weeks at which time physical examination \& ultrasound study was performed.
\end{abstract}

\section{Introduction}

Foam sclerotherapy (FS), as practiced by the author for more than 2 decades, has consisted of the injection
Some patients received sclerotherapy of unsightly spider veins of legs at time of follow-up visits. For better visual understanding of the FWS technique a clip on YouTube.com is available:

https://www.youtube.com/watch?v=Usw9_d2uTvI\&t=4s

Results: The cohort consisted of 823 incompetent veins in 657 patients. 505 patients were female and 152 were male. Mean age was 48.5 (23 - 76). Each case was followed for 12 months. Overall success rate of sclerotherapy in both the FS and FWS groups was similar at around $90 \%$ or above for different groups of veins treated. Overall complication rate was $20.2 \%$ in the FS group versus $6 \%$ in the FWS group.

Discussion: The author presents the results of a retrospective analysis of success and complication rates between the standard method of foam sclerotherapy (FS) of varicose veins, as practiced at his vein clinic, with a modified method named foam washout sclerotherapy (FWS) that he implemented for the first time in late 2008. The author presents that by removing the injected foam from the lumen of targeted varicosity, complication rate is lowered while success rate is identical in both methods.

Conclusion: The author suggests that the modified technique of FWS in the treatment of lower extremity varicose veins is associated with fewer complications compared to FS, is equally as effective as FS and in the future FWS may replace the current FS technique for chemical ablation of some cases of varicose veins of legs.

Keywords Foam Washout Sclerotherapy, Foam Sclerotherapy, Varicose veins, Sclerotherapy Complications, Chemical Vein Ablation

of a small volume (4-7 $\mathrm{ml}$ in most cases) of a detergentbased sclerosing agent into a targeted incompetent varicose 
vein of lower extremity with the goal of complete sclerosis resulting in occlusion. On the average, the success rate of foam sclerotherapy has been reported at around $75.8-87 \%{ }^{1-2}$. However, there have been reports of several complications such as deep venous thrombosis (DVT), migraine headaches, vision disturbances, and strokes ${ }^{1-11}$

The mechanism for Central Nervous System (CNS) symptoms and migraine headaches has been suggested to be related to the release and circulation of vasoactive mediators (endothelins) from injured endothelial cells associated with foam sclerotherapy ${ }^{8,9,12}$.

The author has pioneered and implemented a new method of foam sclerotherapy attempting to lower the rate of the above complications. This new method, Foam Washout Sclerotherapy (FWS), involves removing most to all of the injected foam from the lumen of the targeted varicosity, and potentially removing the endothelins thought to be responsible for some of the complications. Thus, the current paper searches for a possible lowered risk of DVT as well.

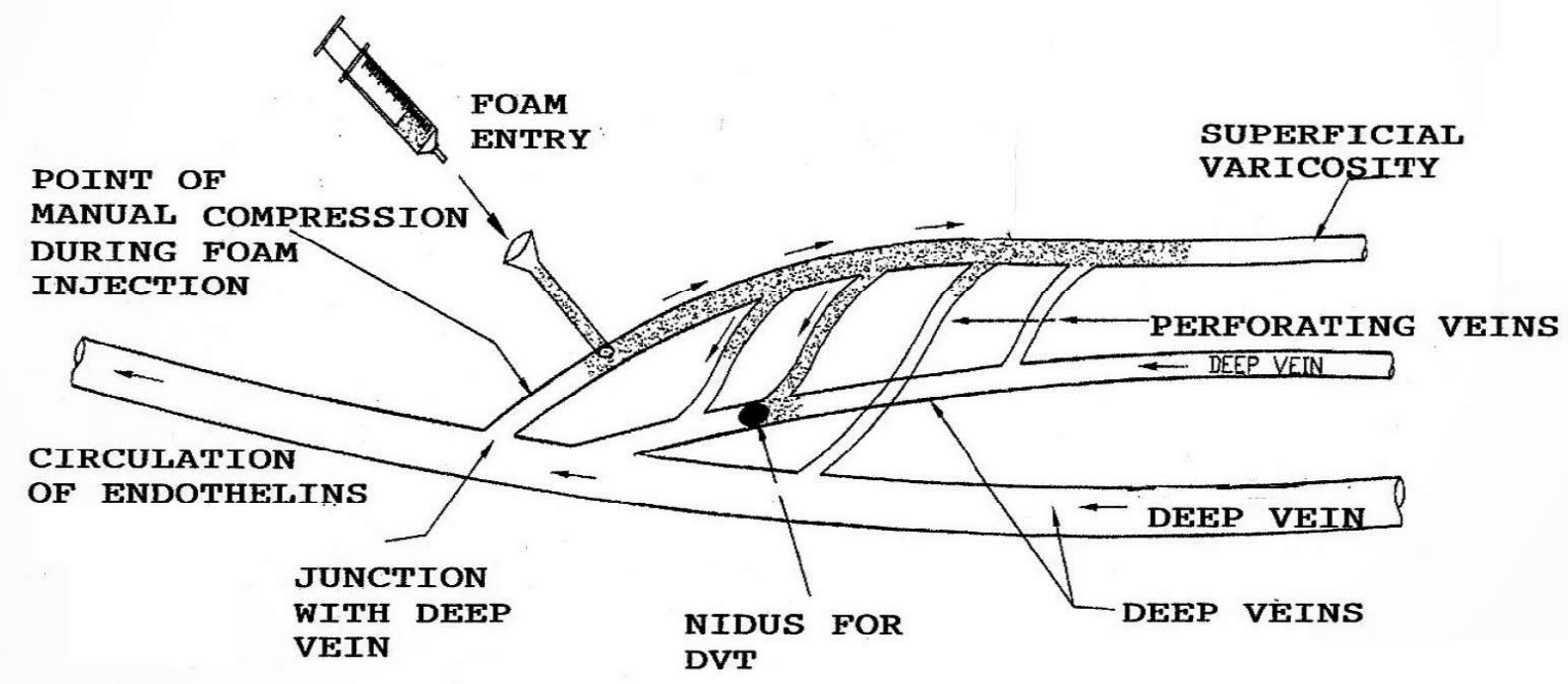

Fig. 1 - Potential for complications in foam sclerotherapy of a superficial varicosity.

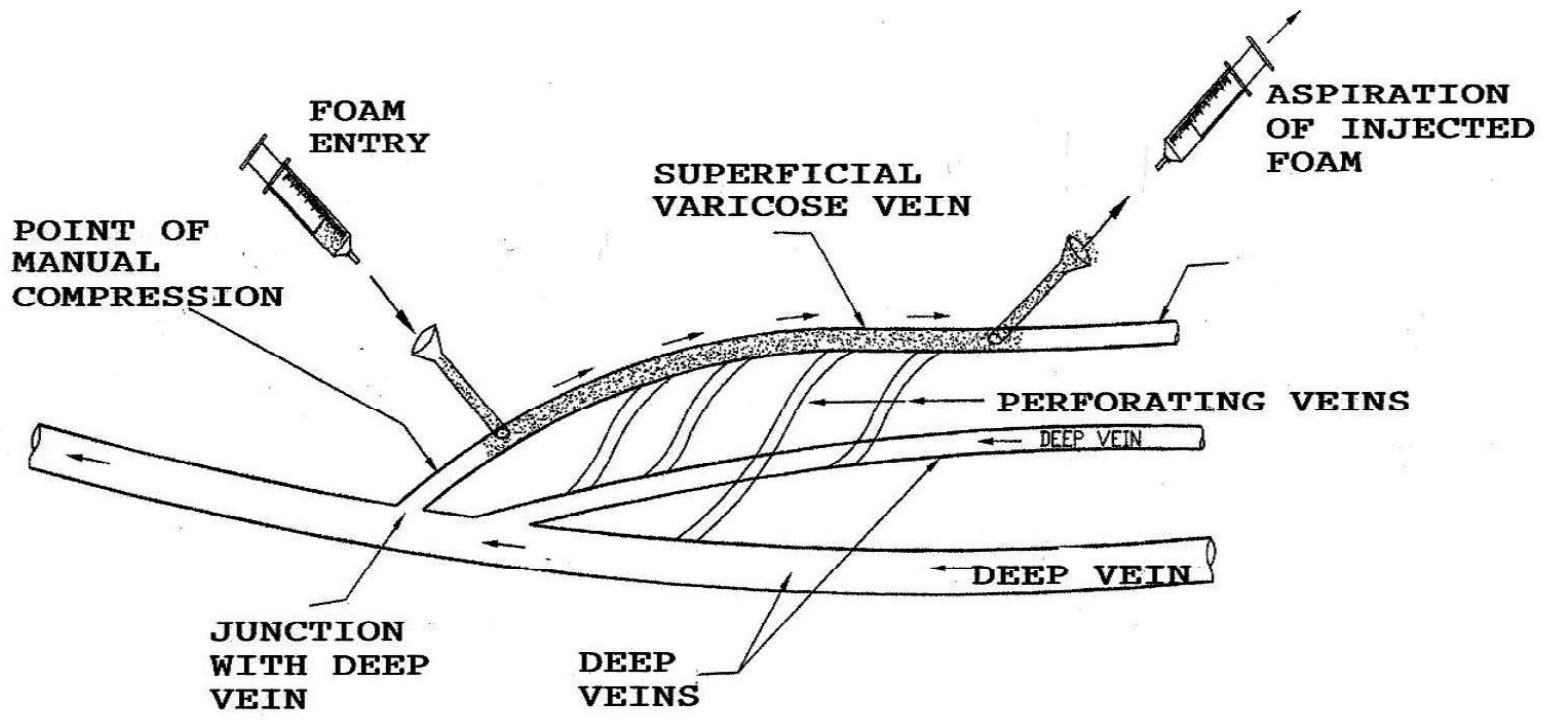

Fig. 2 - Foam Washout Sclerotherapy of superficial varicose veins. 


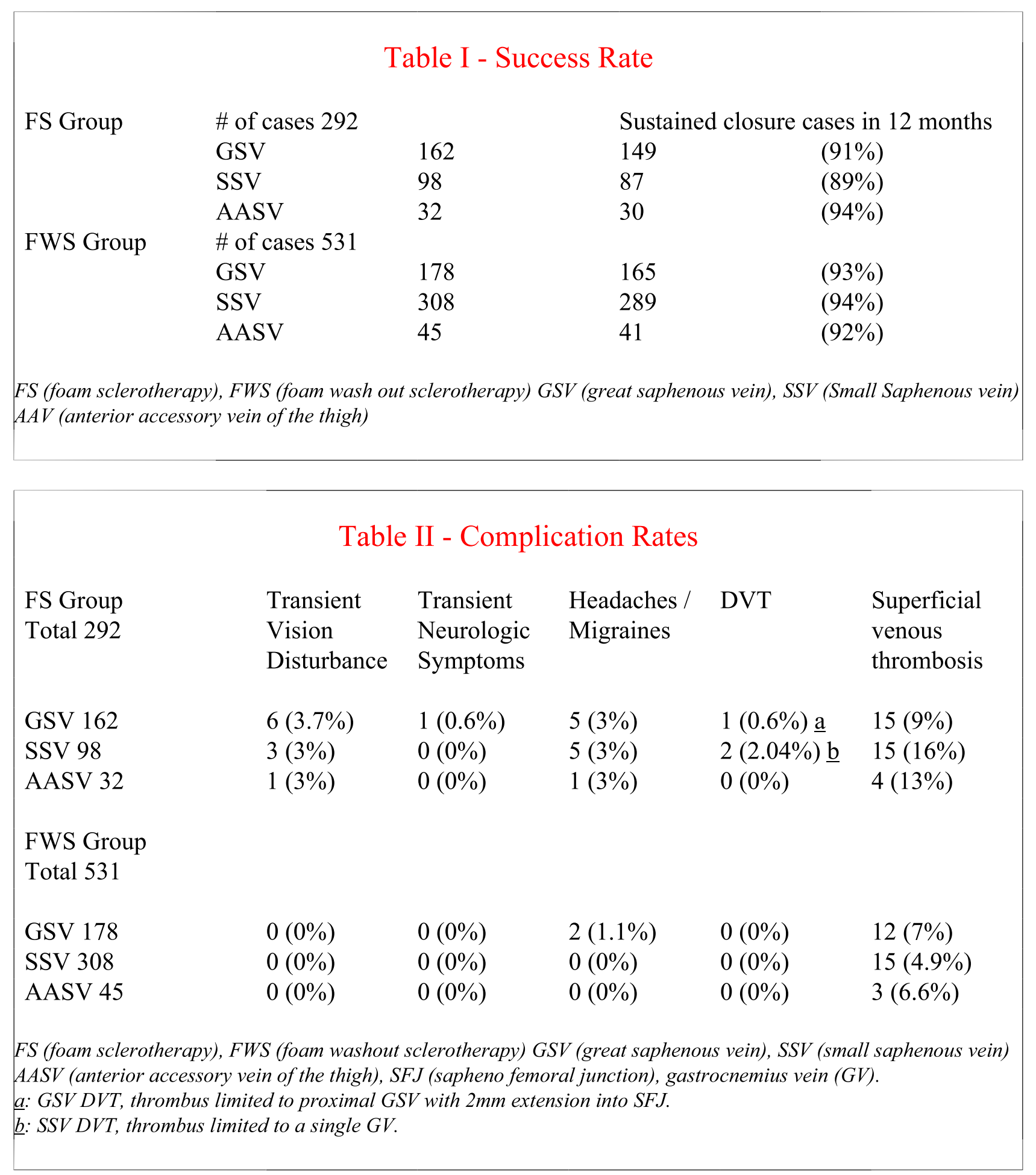

\section{Material and methods}

\section{Techniques}

Foam sclerotherapy (FS) as practiced by the author: After ultrasound/doppler mapping of reflux sources in all cases of varicose veins, foam sclerotherapy begins by injecting the proximal segment of the incompetent vein at
3-5 $\mathrm{cm}$ distal to the source of reflux (junctional reflux or incompetent perforating vein). The patient is placed in the supine position and the leg is elevated 10-20 degrees during the foam injection. Then, while the ultrasound transducer 
is monitoring the appearance of foam in the lumen of the targeted vein, the foamed sclerosing agent is injected over 3-5 seconds. Using the ultrasound transducer, as soon as the foam appears at the reflux source, since the injection is done near the incompetent connection to a deep vein, manual compression is applied there for a few seconds until the injection is completed and catheter is withdrawn to prevent the foam from entering the deep vein system. Foam is prepared by mixture of $\mathrm{CO}_{2}$ gas with either sodium tetradecyl sulfate (STS) or Polidocanol using the Tessari method. The author used STS in concentrations 1.5\%-3\% for varicose veins with a caliber $>7 \mathrm{~mm}$. Polidocanol $1 \%$ is used for veins up to $7 \mathrm{~mm}$ in caliber. Liquid to gas ratio is usually at $1: 3$ or $1: 4$. The foam is allowed to stay in the system and none of the injected material is withdrawn. After the treatment compression stockings are used for 7-14 days. (Figure 1)

The author's modified technique (Figure 2), named foam washout sclerotherapy (FWS) consists of placement of at least two IV access catheters $20 \mathrm{G}-19 \mathrm{G}$ size, with lengths between $3-5 \mathrm{~cm}$ at two levels (approximately 10-12 $\mathrm{cm}$ apart) in the targeted varicose vein segment. The tip of the most proximal catheter, which receives the first foam injection, is approximately $3-5 \mathrm{~cm}$ distal to the identified incompetent perforating vein or junction. To avoid collapse of the lumen of the targeted varicose vein, the leg is kept at body level (the leg is not elevated). Then the ultrasound probe, in transverse orientation, is placed at the source of reflux over the target varicose vein.

Next, injection of 4-7 ml sclerosing foam (STS 1.5\%-3\% for large varicose veins and Polidocanol $1 \%$ for small varicose veins up to $7 \mathrm{~mm}$ in diameter) into the proximal IV catheter is started. The injection is made over 3-4 seconds, while applying pressure over the reflux source using the ultrasound probe to prevent the foam from entering the deep vein system. In the middle of the injection an assistant starts applying a very gentle negative aspiration force to the out-port syringe very slowly at the beginning (to avoid collapsing the vein walls over the tip of the IV catheter) and starts to aspirate the foam and continues this gentle action until withdrawal of foam ceases. Next, while proximal to distal direction massage is applied, both catheters are removed and compression stockings are put on the leg, and the patient is ambulated. Following both methods of sclerotherapy, $30-40 \mathrm{mmHg}$ Compression stockings are used for a period of 7-14 days (14 days for GSV \& SSV reflux cases and 7 days for AASV reflux treatment).

When the incompetent varicosity is longer than 12 centimeters, more than 2 access catheters are placed (A, B, C..) before starting the treatment and the same procedure is repeated in a stepwise fashion starting from the most proximal segment first (A-B, B-C). This modification is named multi-segment FWS.

The aim of the current preliminary paper is to present the FWS procedure. A thorough statistical analysis of FWS data will be included in future articles covering FWS subject.

\section{Patients Characteristics}

Our cohort consisted of 657 patients, 505 of which were female and 166 received treatment on two different varicose veins, making a total of 823 treatments. There were 292 treatments using FS and 531 using FWS. Mean age was $48.5(23-76)$.

The high proportion of SSV cases included in the FWS group is due to the choice of the patients and availability of other treatment options for GSV and SSV disease during the latter part of the study period.

Since this was not a prospective study, no attempts were made to allocate cases in equal numbers between different treatments. Therefore, the total number of incompetent vein types which received either type of treatment technique was not planned. Patient preferences were a major factor in choosing the treatment method.

Patients were followed up for 12 months $(9-14)$ : by phone calls in 24 hours and re-exam and ultrasound evaluation in 2 months, 6 months, and 12 months. Most patients had a follow visit at 2-3 weeks as well. At follow up visits some patients received sclerotherapy of unsightly spider veins. None of the varicose veins treated by FS and FWS methods received retreatment during follow up visits.

Treatment success was defined as: Complete occlusion and lack of reflux in treated varicose veins at both 6 and12 months follow up. Any recanalization that demonstrated reflux was not considered a success.

This retrospective study looked only at success rates in the two groups as defined above and compared complication rates in the two groups without attention to any other factor.

For better visual understanding of the FWS technique a clip on YouTube.com is available:

https://www.youtube.com/watch?v=Usw9 d2uTvI\&t=4s

\section{Results}

In the FS group success rate was $91 \%, 89 \%, 94 \%$ for GSV, SSV, and AASV respectively. The success rate was $93 \%, 94 \%$, and $92 \%$ for GSV, SSV, and AASV respectively for the FWS group. Overall, the success rates are essentially identical (Table 1). 
In the FS group the total combined complication rate for combined complication rate was $20.2 \%$, while the total complication rate in the FWS group was $6 \%$ (Table 2).

There were no cases of DVT in FWS cases. In the FS group one patient treated for SFJ/GSV reflux developed extension of great saphenous vein (GSV) thrombus into the saphenous femoral junction (SFJ) region and two cases of gastrocnemius vein thrombosis (classified as DVT in this series) were found.

There was no case of PE in either group. There were differences in other complication rates between the two methods: vision disturbances, headaches, and superficial venous thrombosis were lower in the FWS compared to the FS group. It may be noteworthy to mention that regarding the very few cases of DVT that were observed, all were in the FS group and 2:3 of the cases involved only part of gastrocnemius veins.

Transient neurologic symptoms was in $0.6 \%$ of FS group and was not noted in FWS group.

\section{Discussion}

FWS has been introduced and implemented by the author in 2008 in a clinical setting, at a time when there were no prior articles covering the FWS technique or its comparison to FS.

A first glance at the complication rate in our FS group may suggest a higher rate of DVT than in some published $\operatorname{articles}^{3,10}$. However, the author points out that no previously published articles have included in their DVT complication rates such things as gastrocnemius vein thrombosis and thrombus extending to the SFJ level without involvement of the femoral vein, as we have done in this paper $^{3}$.

Superficial venous thrombosis is commonly observed in phlebology practice and we recorded this as a complication. Our series recorded superficial venous thrombosis at $4.9 \%$ to $16 \%$ depending on which veins were injected. This compares favorably with one previous article recording a rate of phlebitis of $8.2 \%$ to $33 \%{ }^{2}$.

Our rate of transient headaches after foam sclerotherapy (FS) is similar to published rates4). We regard the overall published rate of major neurological complications is under estimated and more research into actual complication rate is recommended ${ }^{5-7}$. Thus, the author's complication rate in the FS group is similar to published articles. Our complication rate with the new method of foam washout sclerotherapy (FWS) is less than with FS group, while the success rate is similar in both groups.

We consider that the most important factor through which FWS provides safer foam sclerotherapy, appears to be the fact that in the FWS method the application of positive pressure (injection of foam) at the proximal site of injection and negative pressure at the distal foam withdrawal point (out-port) of the same varicose vein provides a desired directional foam flow, which prevents the foam from flowing into unwanted territories such as deep veins through perforating veins ${ }^{3,5-7}$ and adjacent healthy veins. With FWS the treating physician selects exactly the segment to be sclerosed. Furthermore, the absence of any vision disturbance and migraine headaches in FWS suggests that most probably the withdrawing syringe content contains the used foam as well as any immediately released endothelins, which are extracted in place of being allowed to circulate in the patient's circulation $^{8,9}$.

Since the success rate between FS group and FWS group are identical, it appears that 5-10 seconds of contact of the endothelium with an effective concentration of a detergent based sclerosing foam, as is done in FWS, is sufficient to achieve effective chemical ablation of varicose veins (Table II).

If the FWS technique is performed correctly, since the injected foam is removed instead of being left in the circulation system, the treating physician has more freedom over choice of volume and concentration of the injected foam, as well as the contact time of foam with the endothelium of the targeted segment of varicosity. Thus, the FWS may achieve better results in cases of large caliber varicosities.

Future studies comparing FWS with FS and other treatment modalities may provide additional useful information regarding foam sclerotherapy techniques in clinical practice.

\section{Conclusion}

Based on a 4-year single center experience, the author presented the results of a retrospective analysis of success and complication rates of two foam-based chemical ablation methods of treating varicose veins consisting of standard method of foam sclerotherapy (FS), as practiced at his vein clinic, with a modified method named foam washout sclerotherapy (FWS) that he implemented for the first time in late 2008 .

The type of incompetent veins that were included in this article were great saphenous vein (GSV) and small 
saphenous vein (SSV) and anterior accessory saphenous vein (AASV). The study included chemical ablation of 832 incompetent vein cases among 657 individuals. Treatment success was defined as complete occlusion and lack of reflux in treated varicose veins at both 6 and 12 months follow up visits. Any recanalization that demonstrated reflux was not considered a success.

\section{References}

1) Hamahata A, Yamaki T, Sakurai H. Outcomes of UltrasoundGuided Foam Sclerotherapy for Varicose Veins of the Lower Extremities: A Single Center Experience. Dermatol Surg. 2011 Jun 2.

2) Kakkos SK, Bountouroglou DG, Azzam M, Kalodiki E, Daskalopoulos M, Geroulakos G. Effectiveness and safety of ultrasound-guided foam sclerotherapy for recurrent varicose veins: immediate results. J Endovasc Ther. 2006 Jun;13(3):357-64.

3) Guex JJ, Allaert FA, Gillet JL, Chleir F. Immediate and midterm complications of sclerotherapy: report of a prospective multicenter registry of 12,173 sclerotherapy sessions. Dermatol Surg. $2005 \mathrm{Feb} ; 31(2): 123-8$.

4) Rabe E, Pannier F. Sclerotherapy of varicose veins with polidocanol based on the guidelines of the German Society of Phlebology. Dermatol Surg. 2010 Jun;36(Suppl 2):968-75.

5) Bush RG, Derrick M, Manjoney D. Major neurological events following foam sclerotherapy. Phlebology 2008;23(4):189-92.

6) Hahn M, Schulz T, Jünger M. Late stroke after foam sclerotherapy. Vasa. $2010 \mathrm{Feb}$;39(1):108-10.
After analysis of all data it was shown that the success rate was similar in both groups while the overall complication rate was lower in the FWS group. The author presents that by removing the injected foam from the lumen of targeted varicosity, complication rate is lowered while success rate remains identical in both methods.

7) Guex JJ. Complications and side-effects of foam sclerotherapy. Phlebology. 2009 Dec;24(6):270-4.

8) Frullini A, Barsotti MC, Santoni T, Duranti E, Burchielli S, Di Stefano R. Significant endothelin release in patients treated with foam sclerotherapy. Dermatol Surg. 2012 May;38(5):741-7.

9) Frullini A, Felice F, Burchielli S, Di Stefano R. High production of endothelin after foam sclerotherapy: a new pathogenetic hypothesis for neurological and visual disturbances after sclerotherapy. Phlebology 2011 Aug;26(5):203-8.

10) Hill DA. Neurological and chest symptoms following sclerotherapy: A single centre experience. Phlebology 2013 Aug 14; [Epub ahead of print]

11) Cavezzi A , Parsi K. Complications of foam sclerotherapy. Phlebology 2012 Mar;27(Suppl 1):46-51.

12) Weiss MA, Hsu JT, Neuhaus I, Sadick NS, Duffy DM. Consensus for sclerotherapy. Dermatol Surg. 2014 Dec;40(12):1309-18. 